\title{
latrogenic botulism - a possible cause of generalised muscular weakness in child
}

\author{
Mirela Silvia Iancu ${ }^{1,2}$, Luminita Elena Spatariu ${ }^{1,2}$, Victoria Hurduc ${ }^{1,2}$, Doina Anca Plesca ${ }^{1,2}$ \\ ${ }^{1}$ Pediatrics Discipline, "Carol Davila" University of Medicine and Pharmacy, Bucharest, Romania \\ 2"Dr. Victor Gomoiu" Clinical Children's Hospital, Bucharest, Romania
}

\begin{abstract}
latrogenic botulism is a rare but significant complication in botulinic toxin treatment, both in adult or pediatric patient. This article presents the case of a 5 years old child, diagnosed with cerebral palsy, tetraparesis and global development delay, predominant motor delay who, within 1 week after injection with botulinic toxin in lower limbs, developed generalized muscular weakness, difficulties in raising the head and sitting, drooping eyelid, speech and eating disturbances, symptoms that slowly resolved in about 8 weeks.
\end{abstract}

Keywords: botulinum toxin, iatrogenic botulism, cerebral palsy, child

\begin{abstract}
Abbreviations
$\mathrm{BT}=$ botulinum toxin

$\mathrm{GA}=$ gestational age

$\mathrm{CT}=$ computer tomography

$\mathrm{WB}=$ weight at birth

$\mathrm{RSV}=$ respiratory syncytial virus

$\mathrm{AOM}=$ acute otitis media

SFEMG $=$ single-fiber electromyography

GMFCS $=$ the gross motor function

classification system

$\mathrm{CMPA}=$ cow's milk protein allergy

$\mathrm{CP}=$ cerebral palsy
\end{abstract}

\section{INTRODUCTION}

Botulinum toxin (BT) has been used for long time for treatment of different neuromuscular disorders (either at manufacturer-recomandation or in off-label indications) like dystonia, spasticity, spasmodic torticollis, blepharospasm, hemifacial spasm, strabismus. Over the last few years the indications for BT toxin use have extended also for cosmetic applications (rejuvenation therapy and hyperhidrosis), treatment for chronic migraine, sialorrhea, neurogenic and nonneurogenic bladder dysfunction. In our country the use of abobotulinumtoxin A (Dysport) is authorized only for dynamic equines foot deformity in children with cerebral palsy (CP) 2 years of age and older.
The exact dose of botulinum toxin A recommended for treatment of lower limb spasticity in children with cerebral palsy is not clearly specified. This should be individualized and estimated according to size of the muscle, severity of spasticity, previous botulinum toxin injections and patient response or adverse reactions. However, total dose per treatment session should not exceed 10-15 U/kg per limb or 20$30 \mathrm{U} / \mathrm{Kg}$ for bilateral lower limb injections or $1000 \mathrm{U}$, whichever is lower. The total dose administered should be divided between the affected muscles and, when possible, distributed across more injections in a single muscle (1). Repeat treatment should be administered no sooner than 12 weeks after the previous injection. 


\section{CASE PRESENTATION}

A 5-year-old girl diagnosed with cerebral palsy and quadriplegia receive botulinum toxin injection for spasticity of lower limbs. After 1 week she presented with weekness, generalized muscular hypotonia, loss of head control, poor sitting, reduced movements, right palpebral ptosis, dysarthria, difficulty in swallowing, feeding and speech disorder and drooling.

Her mother had an unsurveilled pregnancy and the child was spontaneously delivered at 26-week gestation, weighting 995 grams and with APGAR score of 4 at 1 minute and 8 at 5 minutes. She had intraventricular hemorrhage within the third day of life and severe anemia and received five blood transfusions. When she was 9-day-old she was diagnosed with sepsis and she received antibiotherapy and total parenteral nutrition. Because of post-hemorrhagic internal hydrocephalus she underwent several ventricular punctures and when she was 5 months-old a ventriculoperitoneal shunt was placed. Due to further infection with Pseudomonas and Staphylococcus the shunt was removed. She received antibiotic and antifungic treatment for 1 month and after that a subgaleal shunt was put instead but this also had disfunctions and needed puncture and revision for twice. At 9-monthold subgaleal shunt was replaced by a ventriculoperitoneal shunt with good function. She began massage and physiotherapy since she was an infant, along with nutritional recovery. When she was 15 -month-old she moved to a center for children with special needs. From that moment she was fine, with no complication of neurosurgery and only 2 admissions in the ENT department for acute otitis media (AOM).

She received botulinum toxin injections for the first time when she was 5 year 2 months old. At that moment she was evaluated with severe delay in motor development (GMFCS V), marked spasticity in adductor muscles of the thighs and triceps surae, but was able to sit, to roll over, to stay in hands and knees position, had difficulty to alternate legs. Expressive language was present. She received botulinum toxin injections (dosage unknown) both in upper and lower limbs, well tolerated and without side effects and she continued physiotherapy. After 4 months 1000 UI Dysport were injected into lower limbs only, along with physiotherapy and ergotherapy. One week later she progressively presented with generalized weakness, palpebral ptosys on the right side, feeding troubles with swallow difficulties and disarthria. 12 days after the treatment she had fever and one episode of vomiting, followed by few nasal secretions and mild cough. She had a head CT and neurosurgical examination and shunt related complication was excluded.
She was admitted at an infectious diseases hospital where she underwent a lombar puncture that excluded meningitis and was diagnosed with and treated for respiratory syncytial virus pneumonia. 3 weeks after the injection of botulinum toxin her neuromotor function was re-evaluated. A single-fiber EMG showed increased jitter and synaptic latencies at neuromuscular junction. Based on clinical and electrophysiological findings, a diagnosis of botulism was suspected. Her clinical state improved and symptoms slowly disappeared in about 2 months. She had no botulinum toxin injections after that, she continued her physio and ergotherapy programme.

\section{DISCUSSIONS}

There are 8 distinct serotypes of botulinum toxins (A, B, C1, C2, D, E, F and G) produced by Clostridium botulinum, an anaerobic Gram-positive bacilli, recognized as the causative agent of botulism since 1897 (2). They have different pharmacological properties and only two serotypes are available for therapeutic clinical use: botulinum toxin type A, the most widely used worldwide, and the less used one, type B.

Botulinum toxin acts at the presynaptic cholinergic nerve terminals where inhibits the release of acetylcholine into the synaptic cleft, thus preventing muscle contraction (chemodenervation) (Fig. 1). The effect lasts for about 3 months and the return of synaptic activity rely on plasticity and remodeling, with sprouting (new but transitory nerve processes which form functional synapses until the original nerve terminals regenerate (3).

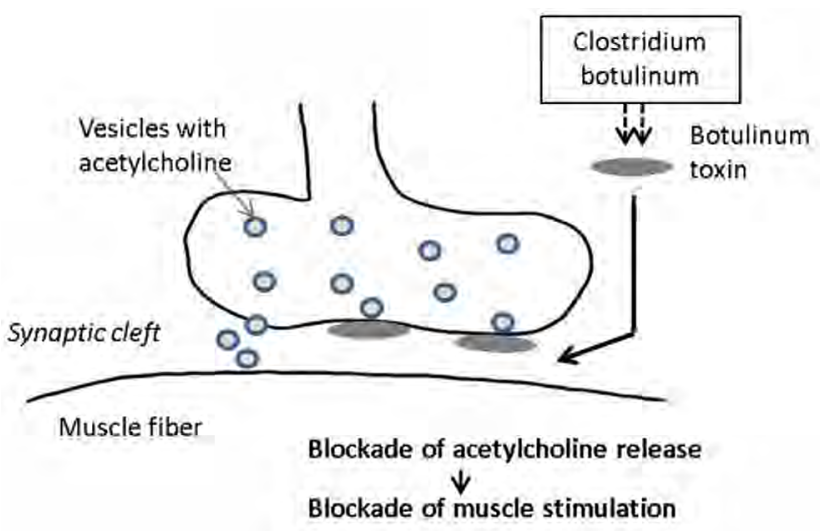

FIGURE 1. Mechanism of action of botulinum toxin

Botulinum toxin is a safe and well-tolerated treatment (4), with few side effects, generally mild and transitory, like local pain or infections of upper respiratory tract, with nasal obstruction, rhinorrhea, sore throat, cough and fever. Systemic effects, like anaphylaxis or flu-like symptoms are rare and maybe im- 
mune mediated. However, systemic toxin spread can lead to iatrogenic botulism, a sub-form of botulism, potentially fatal, which manifests with general muscle weakness, velo-pharyngeal insufficiency and dysarthria, dysphonia, dysphagia, eyelid ptosis, diplopia, respiratory distress, urinary incontinence. The mechanism responsible for the generalized diffusion of botulinum toxin is not known but both systemic spread and retrograde axonal spread into the central nervous system are possible. Side effects of toxin could be related more to the total injection dose than to injection dose based on body weight (5). Interestingly, systemic side effects can occur after repeated injections but also after the first administration of botulinum toxin, after therapeutic doses but also al lower doses, within few hours or weeks following injection, making difficult to predict patients at potential risk (6). There were situations when treatment with botulinum toxin was interrupted or was continued and patient didn't show remote severe adverse effects. Effect of botulinum toxin in patients with neuromuscular disorders or treated with aminoglycosides may be potentiated and should be closely monitored. This treatment is contraindicated for patients known to have allergies to cow's milk protein or hypersensitivities to botulinum toxin preparations.

Electrophysiological studies represent a useful support for confirming the diagnosis of iatrogenic botulism although there are only few and heterogenous reports in the medical literature. There is no specific pattern of electrophysiological abnormalities in iatrogenic botulism and the diagnosis is based on clinical findings and anamnestic data but still, acute denervation changings are the most suggestive (7).

BT is generally well tolerated in children and fewer cases of iatrogenic botulism are reported (8). In 882 children treated with BT for cerebral palsy spasticity side effect were mainly respiratory tract infections, bronchitis, pharyngitis and less muscle weakness and falls (9). Willis et al. (10) found no statistical signifi-

\section{REFERENCES}

1. https://reference.medscape.com/drug/dysportabobotulinumtoxina-999220.

2. Nigam PK, Nigam A. Botulinum toxin. Indian J Dermatol. 2010; 55(1):8-14

3. de Paiva A, Meunier FA, Molgo J, Aoki KR, Dolly JO. Functional repair of motor endplates after botulinum neurotoxin type A poisoning: biphasic switch of synaptic activity between nerve sprouts and their parent terminals. Proc Natl Acad Sci USA. 1999;96(6):3200-5.

4. Nauman M, Albanese A, Heinen F, Molenaers G, Relja M. Safety and efficacy of toxin type A following longterm use. Eur J Neurol. 2006; 13(Suppl. 4): 35-40.

5. Bakheit AM, Severa S, Cosgrove A, Morton R, Roussounis SH, Doderlein $L$ Safety profile and efficacy of botulinum toxin $A$ (Dysport) cance differences in adverse effects rates after higher or lower dose injections with BT in children with $\mathrm{CP}$, regardless of etiology, clinical phenotype, severity, functional ability or medication use, unlike Swinney et al. who showed that children in GMFCS levels IV and $\mathrm{V}$ were at increased risk of systemic adverse events (11). Data in 758 patients, mean age 7.2 years, who received a total of 1,594 injections with BT mainly for spastic cerebral palsy showed that doses higher than 1,000 UI didn't improve the therapeutic response and increased the risk of adverse events (5). Actually, randomized controlled trials didn't report severe or long term adverse effects after BT, these were rather found from spontaneously reported cases (12-14).

\section{CONCLUSIONS}

The patient described here has similarities with other reported cases in the literature, about remote effects after BT injections. This girl received BT injections in both lower limbs and 1 week later she started to develop generalised weakness of muscles distant to injection site, with relaxing anticholinergic effects (oropharyngeal and eye related) but no respiratory compromise. Her symptoms resolved after 2 months without treatment. The particularity of this case was that the patient associated a lower respiratory tract infection, reported also as a possible rare side effect after BT injection. For children in GMFCS level V, with severe hypertonia, in whom spasticity represents only one component of the disease, indications of treatment with BT targeted to the focal tonus are more difficult to establish and results are less predictable. Despite the rare but unexpected occurrence of such adverse events due to BT, a careful follow-up of patients receiving this treatment is necessary. Also, it would be useful that parents or carers be alerted to the possibility of these side effects.

Conflict of interest: none declared Financial support: none declared

in children with muscle spasticity. Dev Med Child Neurol. 2001; 43(4):234-238.

6. Phadke CP, Balasubramanian CK, Holz A, et al. Adverse clinical effects of botulinum toxin intramuscular injections for spasticity. Can J Neurol Sci. 2016;43(2):298-310.

7. Leonardi L, Haggiag S, Petrucci A, Lispi L. Electrophysiological abnormalities in iatrogenic botulism: Two case reports and review of the literature. J Clin Neurosci. 2019;60:138-141.

8. Delgado MR, Tilton A, Russman B, Benavides O, Bonikowski M, Carranza J, Dabrowski E, Dursun N, Gormley M, Jozwiak M, Matthews D, Maciag-Tymecka I, Unlu E, Pham E, Tse A, Picaut P. AbobotulinumtoxinA for Equinus Foot Deformity in Cerebral Palsy: A Randomized Controlled Trial. Pediatrics. 2016;137(2):e20152830. 
9. Albavera-Hernandez C, Rodriguez JM, Idrovo AJ. Safety of botulinum toxin type $A$ among children with spasticity secondary to cerebral palsy: a systematic review of randomized clinical trials. Clinical Rehabilitation 2009;23(5):394-407.

10. Willis AW, Crowner B, Brunstrom JE, Kissel A, Racette BA. High dose botulinum toxin $A$ for the treatment of lower extremity hypertonicity in children with cerebral palsy. Dev Med Child Neurol. 2007; 49(11):818-822.

11. Swinney CM, Bau K, Burton KLO, O'Flaherty SJ, Bear NL, Paget SP. Severity of cerebral palsy and likelihood of adverse events after botulinum toxin A injections. Dev Med Child Neurol. 2018; 60(5):498-504
12. Crowner BE, Torres-Russotto D, Carter AR, Racette BA. Systemic weakness after therapeutic injections of botulinum toxin a: a case series and review of the literature. Clin Neuropharmacol. 2010; 33(5):243-247.

13. Bhatia KP, Münchau A, Thompson PD, et al. Generalised muscular weakness after botulinum toxin injections for dystonia: a report of three cases. Journal of Neurology, Neurosurgery \& Psychiatry 1999;67:90-93.

14. Yiannakopoulou E. Serious and long-term adverse events associated with the therapeutic and cosmetic use of botulinum toxin. Pharmacology. 2015;95(1-2):65-9. 